the index date. Fully adjusted models included chronic kidney disease, Charlson comorbidity index, glucocorticoids, and cardiovascular medication use assessed at the time of SLE diagnosis.

Results We identified 290 SLE cases who died and 502 matched controls among 792 individuals with SLE. The mean age at index date was 65.6 years for cases and 64.7 years for controls. The majority were female $(87.9 \%$ of cases and 91.4\% of controls). The mean SLE disease duration was 5.3 years for both groups. Adjusted odd ratios (ORs) for all-cause mortality relative to the remote users were 0.35 (95\% CI: $0.20,0.59$ ) for current users and 3.78 (95\% CI: 2.07, 6.91) for subjects who recently discontinued HCQ (table 1). HCQ non-users had the same risk of death as remote users (OR 0.93 [95\% CI: $0.59,1.44])$. Similar trends were seen for the risk of mortality due to CVD.

Conclusions In this study, we found a nearly four-fold increased risk of death associated with recent HCQ discontinuation and a substantially increased risk of CVD death. This could be partially explained by a direct protective effect of HCQ that is rapidly lost following discontinuation. We also demonstrated a $65 \%$ reduced risk of death among current HCQ users compared with remote users. By leveraging remote

users as the comparison group, we reduced the potential for confounding by indication.

Funding Source(s): CIHR (Grants MOP 125960 and THC 135235).

\title{
300 INSIGHTS FROM SINGLE-CELL RNA SEQUENCING OF SKIN AND KIDNEY IN LUPUS NEPHRITIS
}

${ }^{1}$ Evan Der*, ${ }^{2}$ Hemant Suryawanshi, ${ }^{2}$ Pavel Morozov, ${ }^{2}$ Manjunath Kustagi, ${ }^{3}$ Beatrice Goilav, ${ }^{3}$ Saritha Ranabothu, ${ }^{4}$ Peter Izmirly, ${ }^{4}$ Robert Clancy, ${ }^{4}$ Michael Belmont, ${ }^{5}$ Mordecai Koenigsberg, ${ }^{1}$ Michele Mokrzycki, ${ }^{5}$ Helen Rominiecki, ${ }^{5}$ Jay Graham, ${ }^{5}$ Juan Rocca, ${ }^{4}$ Nicole Bornkamp, ${ }^{1}$ Nicole Jordan, ${ }^{1}$ Emma Schulte, ${ }^{4}$ Ming Wu, ${ }^{5}$ James Pullman, ${ }^{6}$ Kamil Slowikowski, ${ }^{6}$ Soumya Raychaudhuri, ${ }^{7}$ Joel Guthridge, ${ }^{7}$ Judith A James, ${ }^{4}$ Jill Buyon, ${ }^{2}$ Thomas Tuschl, ${ }^{8}$ Chaim Putterman. ${ }^{1}$ Albert Einstein College of Medicine; ${ }^{2}$ The Rockefeller University; ${ }^{3}$ Children's Hospital at Montefiore; ${ }^{4}$ NYU School of Medicine; ${ }^{5}$ Montefiore Medical Center; ${ }^{6}$ Broad Institute; ${ }^{7}$ Oklahoma Medical Research Foundation; ${ }^{8}$ Albert Einstein College of Medicine and Montefiore Medical Center

\subsection{6/lupus-2019-Ism.300}

Background Classification and treatment decisions in lupus nephritis (LN) are largely based on renal histology. Single-cell

C

A

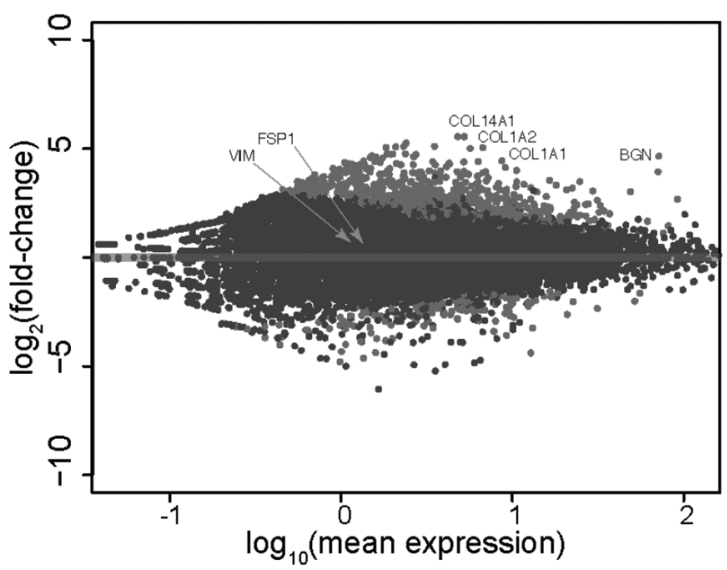

B

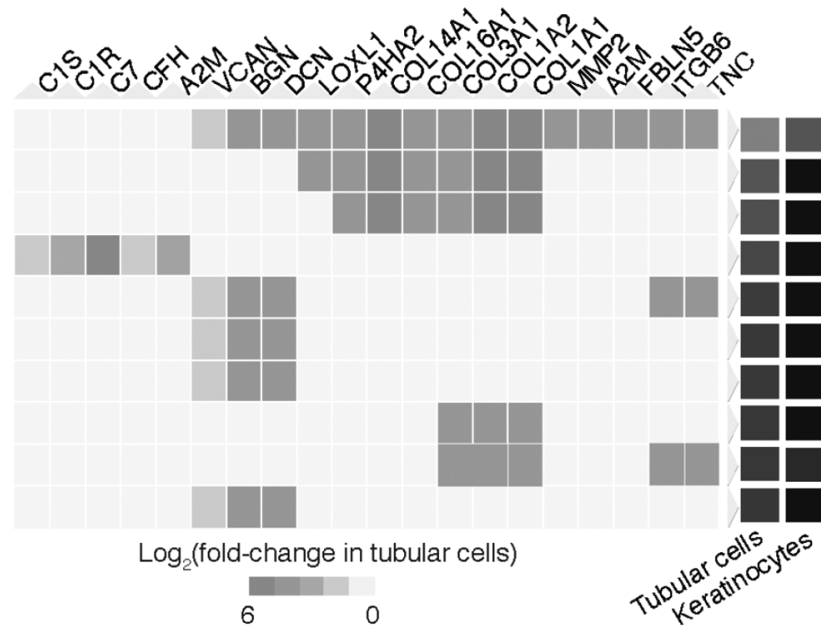

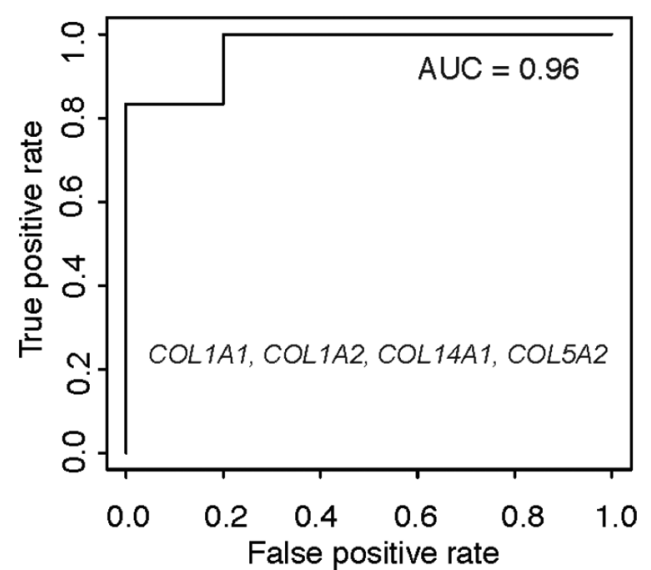

\section{Enriched Terms}

Extracellular matrix organization

Collagen formation

Collagen biosynthesis and modifying enzymes

Complement and coagulation cascades

ECM proteoglycans

CSIDS degradation

Dermatan sulfate biosynthesis

Scavenging by Class A Receptors

ECM-receptor interaction

Defective CHST3 causes SEDCJD

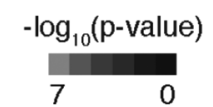

Abstract 300 Figure 1 A fibrotic gene signature as a potential prognostic marker for patients non-responsive to treatment. A) MA plot of differential expression analysis performed between tubular cells of patients responsive $(n=13)$ or non-responsive to treatment $(n=5)$. Significantly differentially expressed genes are colored in red. B) Pathway enrichment analysis of genes identified as upregulated in patients non-responsive to treatment. -Log10(p-value) of each pathway is shown for both keratinocytes and tubular cells colored from least significant (black) to most significant (red). Log2 fold change in gene expression between patients non-responsive to treatment compared with patients responsive to treatment in each pathway is indicated for tubular cells from smallest (grey) to highest (orange). C) Receiver operating characteristic curve of the logistic regression equation of differentially expressed fibrotic genes, COL1A2, COL1A1, COL14A1, COL5A2, with area under the curve (AUC) indicated. 
RNA sequencing (scRNAseq) analysis may accurately differentiate types of renal involvement at the transcriptomic level, and better inform treatment decisions and prognosis.

Methods scRNAseq was performed on kidney and non-lesional skin tissue collected from 20 SLE patients undergoing a clinically indicated renal biopsy. Cell types were determined using principal component analysis and t-distributed stochastic neighbor embedding ( $\mathrm{tSNE}$ ) plotting, resulting in the definitive identification of keratinocytes, tubular cells, mesangial cells, fibroblasts, endothelial cells, and leukocytes.

Results LN patients expressed upregulated IFN response genes in both their tubular cells and keratinocytes. This IFN response signature in tubular cells predicted poor response to therapy 6 months post-biopsy. Tubular cells of non-responder patients also expressed upregulated extracellular matrix proteins and fibrotic markers (figure $1 \mathrm{~A}$ and $1 \mathrm{~B}$ ). Using logistic regression analysis, a 4-gene tubular fibrosis score was created and able to predict response to treatment with an area under curve of 0.9 (figure 1C). Keratinocytes of non-responders also upregulated certain extracellular matrix genes and this response was not observed in peripheral blood mononuclear cells. Differential expression analysis between histology classes indicated an upregulation of IFN and TNF signaling in the tubular cells of patients with proliferative LN compared with membranous.

Conclusions scRNAseq from $2-10 \mathrm{~mm}$ of renal biopsy tissue in SLE can differentiate between the different classes of LN, and provide important insights into potential pathogenic mechanisms. Further, changes in the skin of LN patients can provide a useful source of biomarkers and may reflect important information concerning concurrent kidney pathological events.

Funding Source(s): This work was supported by the Accelerating Medicines Partnership (AMP) in Rheumatoid Arthritis and Lupus Network. AMP is a public-private partnership (AbbVie, Arthritis Foundation, Bristol-Myers Squibb, Foundation for the National Institutes of Health, Lupus Foundation of America, Lupus Research Alliance, Merck Sharp and Dohme, National Institute of Allergy and Infectious Diseases, National Institute of Arthritis and Musculoskeletal and Skin Diseases, Pfizer, Rheumatology Research Foundation, Sanofi, and Takeda Pharmaceuticals) created to develop new ways of identifying and validating promising biological targets for diagnostics and drug development. Funding was provided through grants from the National Institutes of Health (UH2-AR067676, UH2-AR067677, UH2AR067679, UH2-AR067681, UH2-AR067685, UH2-AR067688, UH2-AR067689, UH2-AR067690, UH2-AR067691, UH2AR067694, and UM2-AR067678).

\section{NOVELMIRNA-25 INHIBITS AMPD2 IN PERIPHERAL BLOOD MONONUCLEAR CELLS OF PATIENTS WITH SYSTEMIC LUPUS ERYTHEMATOSUS AND REPRESENTS A PROMISING NOVEL BIOMARKER}

\footnotetext{
${ }^{1}$ Huijing Wang*, ${ }^{2}$ Shuang Ye. 'Department of Rheumatology, South Campus, Renji Hospital, School of Medicine, Shanghai Jiaotong University, Shanghai, China; ${ }^{2}$ Department of Rheumatology, South Campus, Ren Ji Hospital, School of Medicine, Shanghai Jiao Tong University
}

10.1136/lupus-2019-Ism.301
Background Systemic lupus erythematosus (SLE) is a multisystemic autoimmune disease with various clinical manifestations. MicroRNAs (miRNAs) and immunometabolism are recognized as key elements in SLE pathogenesis; however, the relationship between miRNAs in peripheral blood mononuclear cells (PBMCs) and metabolism in SLE remains unclear.

Methods We detected PBMC miRNA and mRNA profiles from 3 pooled SLE patients and 3 healthy controls (HCs) using next-generation sequencing, predicted miRNA targets in dysregulated mRNAs, predicted functions and interactions of differentially expressed genes using bioinformatics analysis, validated candidate miRNAs using qRT-PCR, and investigated the association between the expression of candidate miRNAs and SLE clinical characteristics. Moreover, we validated the direct and transcriptional regulatory effect of NovelmiRNA-25 on adenosine monophosphate deaminase 2 (AMPD2) using a dual-luciferase reporter assay and western blot and confirmed AMPD2 mRNA and protein expression in PBMCs using qRT-PCR and western blot, respectively.

Results Multilayer integrative analysis of microRNA and mRNA regulation showed that 10 miRNAs were down-regulated and 19 miRNAs were up-regulated in SLE patient PBMCs compared with HCs. Bioinformatics analysis of regulatory networks between miRNAs and mRNAs showed that 19 miRNAs were related to metabolic processes. Two candidate miRNAs, NovelmiRNA-25 and miR-1273h-5p, which were significantly increased in the PBMCs of SLE patients $(\mathrm{p}<0.05)$, represented diagnostic biomarkers with sensitivities of $94.74 \%$ and $89.47 \%$, respectively (area under the curve $=0.574$ and 0.788 , respectively). NovelmiRNA-25 expression in PBMCs was associated with disease activity in SLE patients, in both active and stable groups $(\mathrm{p}<0.05)$. NovelmiRNA-25 overexpression downregulated AMPD2 expression in HEK293T cells through direct targeting of the AMPD2 3'UTR $(\mathrm{p}<0.01)$, while inhibition of NovelmiRNA25 activity led to increased AMPD2 expression $(\mathrm{p}<0.01)$. NovelmiRNA-25 overexpression also downregulated AMPD2 protein expression in HEK293T cells; AMPD2 protein expression in SLE patient PBMCs was decreased. Our results show that differentially expressed miRNAs play an important role in SLE.

Conclusions Our data demonstrate a novel mechanism in SLE development that involves the targeting of AMPD2 expression by NovelmiRNA-25. miRNAs may serve as novel biomarkers for the diagnosis and evaluation of disease activity of SLE and represent potential therapeutic targets for this disease.

Funding Source(s): Zhejiang Science and Technology Bureau (No.2012C33126 and 2016 C33214), the National Key Research and Development Program of China (No.2016YFC0903902) 